\title{
CORRIGENDUM
}

\section{USP21 deubiquitylates Nanog to regulate protein stability and stem cell pluripotency}

Xingyu Liu, Yuying Yao, Huiguo Ding, Chuanchun Han, Yuhan Chen, Yuan Zhang, Chanjuan Wang, Xin Zhang, Yiling Zhang, Yun Zhai, Ping Wang, Wenyi Wei, Jing Zhang and Lingqiang Zhang

Signal Transduction and Targeted Therapy (2017) 2, 16046; doi:10.1038/sigtrans.2016.46; published online 3 February 2017

Correction to: Signal Transduction and Targeted Therapy (2016) 1, e16024; doi:10.1038/sigtrans.2016.24; Published online 04 November 2016.

Since the publication of this Research Article, the authors have noticed some of the western blot data in the Figures $1 \mathrm{~b}$ and $\mathrm{c}$ and $5 \mathrm{~d}$ were incorrect. We have now repeated the entire experiments and correct data are provided in this Corrigendum. All of the key findings in this Article are not affected by these corrections.

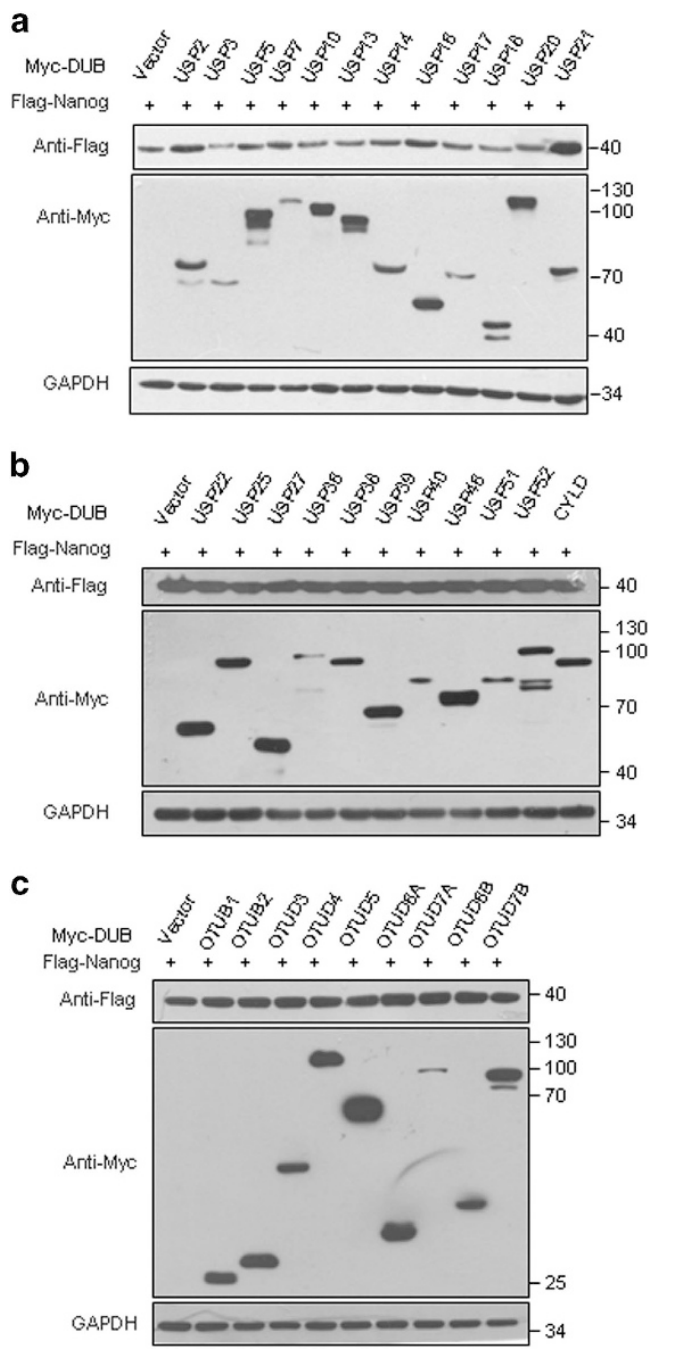

Figure 1. The deubiquitylase screen identifies USP21 as a candidate for Nanog regulation. (a, b) Flag-Nanog and the indicated USP subfamily DUBs were co-transfected into HEK293T cells. Forty-eight hours later, cell lysates were subjected to western blot. (c) FlagNanog and the indicated OTU subfamily DUBs were co-transfected into HEK293T cells. Forty-eight hours later, cell lysates were subjected to western blot. 
a

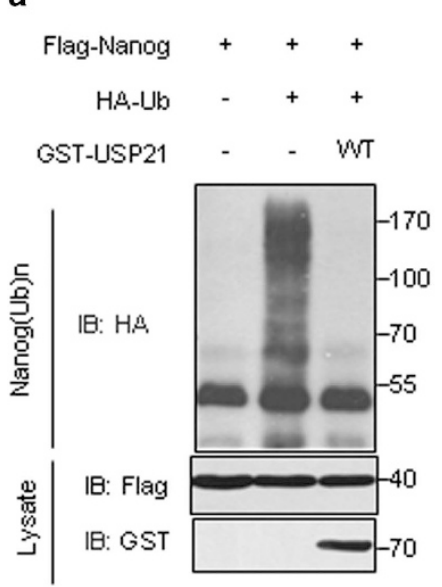

c

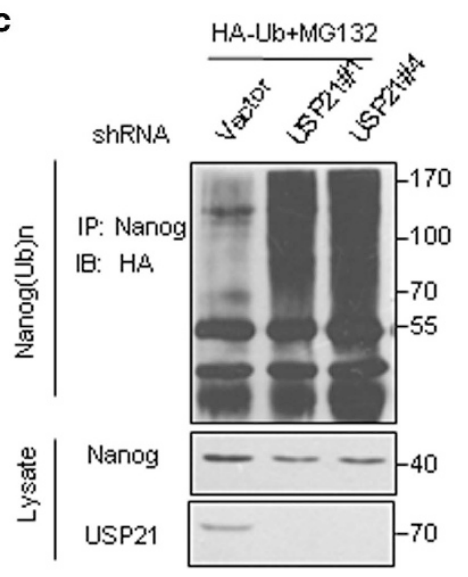

b

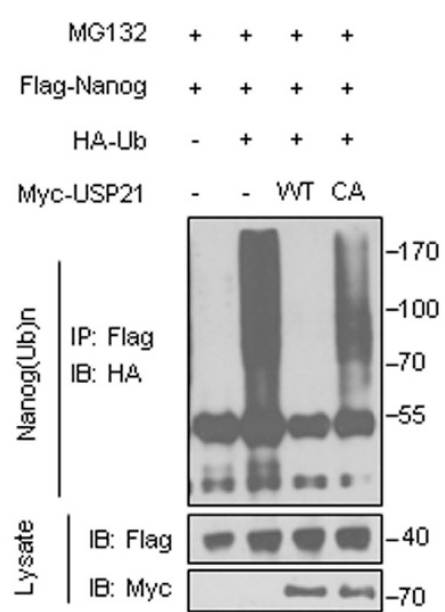

d $\quad H A-I$

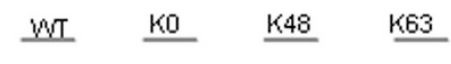

Flag-Nanog ++++++++

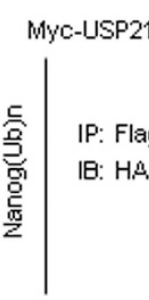

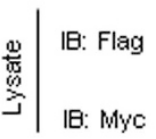
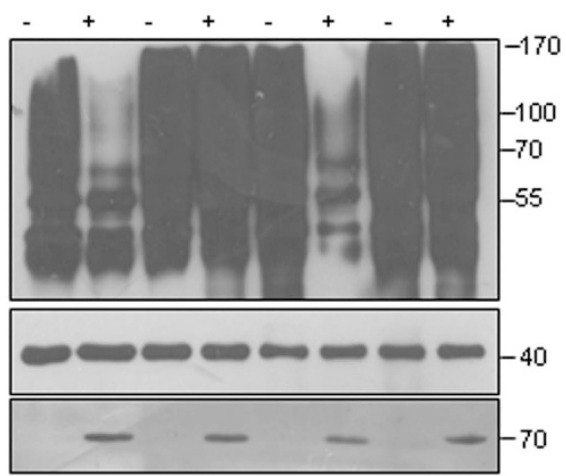

Figure 5. USP21 deubiquitylates Nanog and efficiently removes the K48-linkage type of polyubiquitin chain. (a) Flag-Nanog and HA-ubiquitin were co-expressed in HEK293T cells. After MG132 (10 $\mu \mathrm{M})$ treatment for $8 \mathrm{~h}$, the ubiquitylated proteins was purified by immunoprecipitation with anti-Flag antibodies. GST-USP21 protein purified from E. coli and the ubiquitylated Nanog were mixed and incubated in elution buffer. Then, the mixtures were analyzed via western blotting. (b) HEK293T cells transfected with the indicated constructs were treated with MG132 for $8 \mathrm{~h}$ before collection. The whole-cell lysate was subjected to immunoprecipitate with anti-Flag and western blot with anti-HA. (c) NCCIT cells transfected with the indicated shRNA were treated with MG132 for $8 \mathrm{~h}$ before collection. Nanog was immunoprecipitated with antiNanog and immunoblotted with anti-HA. (d) The Nanog ubiquitylation linkage was analysed in HEK293T cells transfected with Nanog, USP21 and the indicated ubiquitin Lys 0 , Lys 48-only or Lys 63-only plasmids. 